\title{
Engineering Insight for Humanoid Robotics Emotions and Violence with Reference to "System Error 1378"
}

\author{
Sadique Shaikh* \\ Department of Robotics, Institute of Management \& Science (IMS), India
}

Submission: March 03, 2018; Published: June 27, 2018

*Corresponding author: Sadique Shaikh Md, Department of Robotics, Institute of Management \& Science (IMS), Sakegaon-Bhusawal, M.S, India, Email: sids_nsk@rediffmail.com

\begin{abstract}
What kinds of social relationships can people have with computers are there activities that computers can engage in that actively draw people into relationships with them. What are the potential benefits to the people who participate in these human-computer relationships? To address these questions researchers introduces a theory of Relational Agents, which are computational artifacts designed to build and maintain long-term, social-emotional relationships with their users. These can be purely software humanoid animated agents--as developed in this work but they can also be non-humanoid or embodied in various physical forms, from robots, to pets, to jewelry, clothing, hand-held's, and other interactive devices. Central to the notion of relationship is that it is a persistent construct, spanning multiple interactions; thus, Relational Agents are explicitly designed to remember past history and manage future expectations in their interactions with users [1]. Finally, relationships are fundamentally social and emotional, and detailed knowledge of human social psychology with a particular emphasis on the role of affect--must be incorporated into these agents if they are to effectively leverage the mechanisms of human social cognition in order to build relationships in the most natural manner possible. People build relationships primarily through the use of language, and primarily within the context of face-to-face conversation. Embodied Conversational Agents--anthropomorphic computer characters that emulate the experience of face-to-face conversation--thus provide the substrate for this work, and so the relational activities provided by the theory will primarily be specific types of verbal and nonverbal conversational behaviors used by people to negotiate and maintain relationships. This article is also intend if level of Artificial Intelligence reach over Natural Intelligence (Human Intelligence), what would be happen, if System Error 1378 (AI malfunction error) occur one day i.e. robotic violence due to human like emotion in Robots/Humanoid [2]
\end{abstract}

Keywords: Humanoid; Robotics Emotions; Robotics Violence; System Error 1378

\section{Introduction}

Humans take a certain posture in their communication. Can take example, when mankind are happy or sad, cheerful, take a posture in which the activities and behaviour showing through body language like moving and open arms etc. When they are angry, they square the shoulders. When they are tired or sadness, they shrug the shoulder or close the arms. That's why; the emotion and mental condition are closely related to the human posture, gestures, facial expressions and behavior exhibits through body language. And, human obtain many information from partner's posture in their communication [3]. In this situation, the human arms play an important role. Figure $1 \& 2$ below shows the emotional expression exhibited by WE-4RII [4].

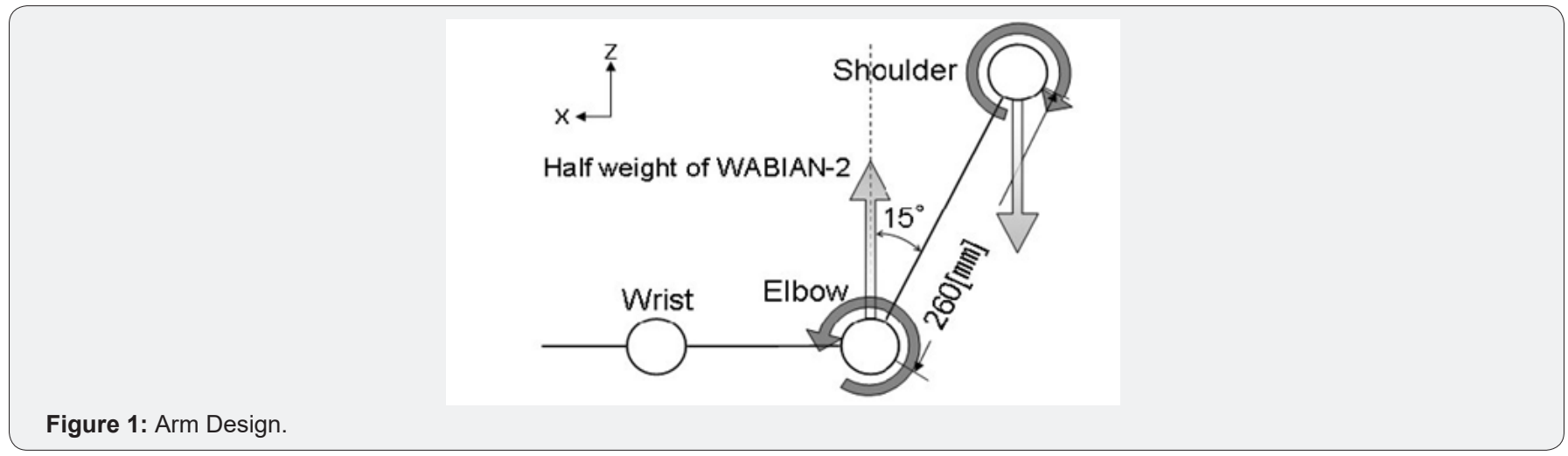




\section{Robotics \& Automation Engineering Journal}

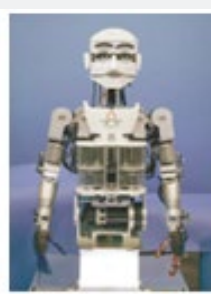

(a) Neutral

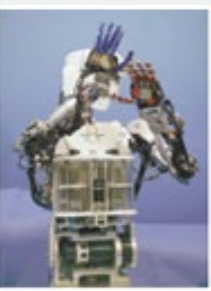

(b) Disgust

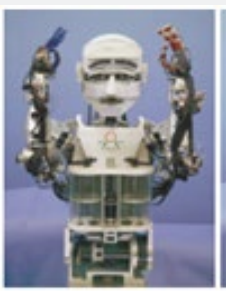

(c) Fear

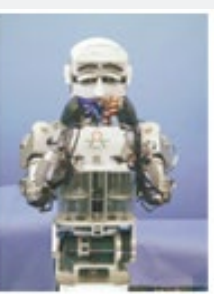

(d) Sadness

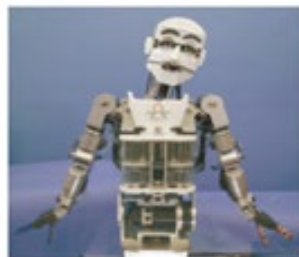

(e) Happiness

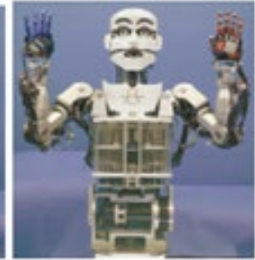

(f) Surprise

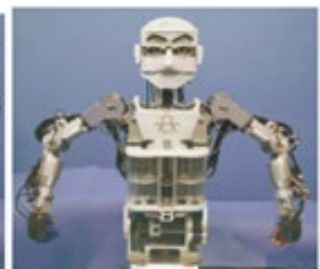

(g) Anger

Figure 2: Robotic expressions.

We can control the generally 6-DOFs (Degree of Freedom) robotic arms' from its tip position precisely as like human's arm. But, all their joint angles are fixed according to the inverse kinematics. By the way, humans have 7-DOFs arms consisting of 3-DOFs shoulder, 1-DOF elbow and 3-DOFs wrist. However, it's considered that there is a rotational center in the base of shoulder joint, and the shoulder joint itself can moves up and down positions as well as moves to and fro, back \& forth so that humans square and shrug their shoulders. We considered that these motions played a very important role in the emotional expressions. Therefore, researchers trying to develop more emotional expressive human-like body movements, gestures feelings, love and affection response supportive movements in humanoid robot arms than the usual 6-DOFs robot arms [5].

\section{Robotic Software Architecture Model}

(Figure 3) Risk assessment is an interdisciplinary subject, which runs together psychological, ethical, legal, and economic considerations. A major problem in risk assessment is the confusion between popular concepts of risk from robots (the 'subjective risk'), which has largely been made irrational by the various fictional depictions autonomous robots destroying humankind and running amok (as in Terminator and I, Robot, among many other movies) and the actual objective risk of deploying robots, i.e., what rational basis is there for worry?

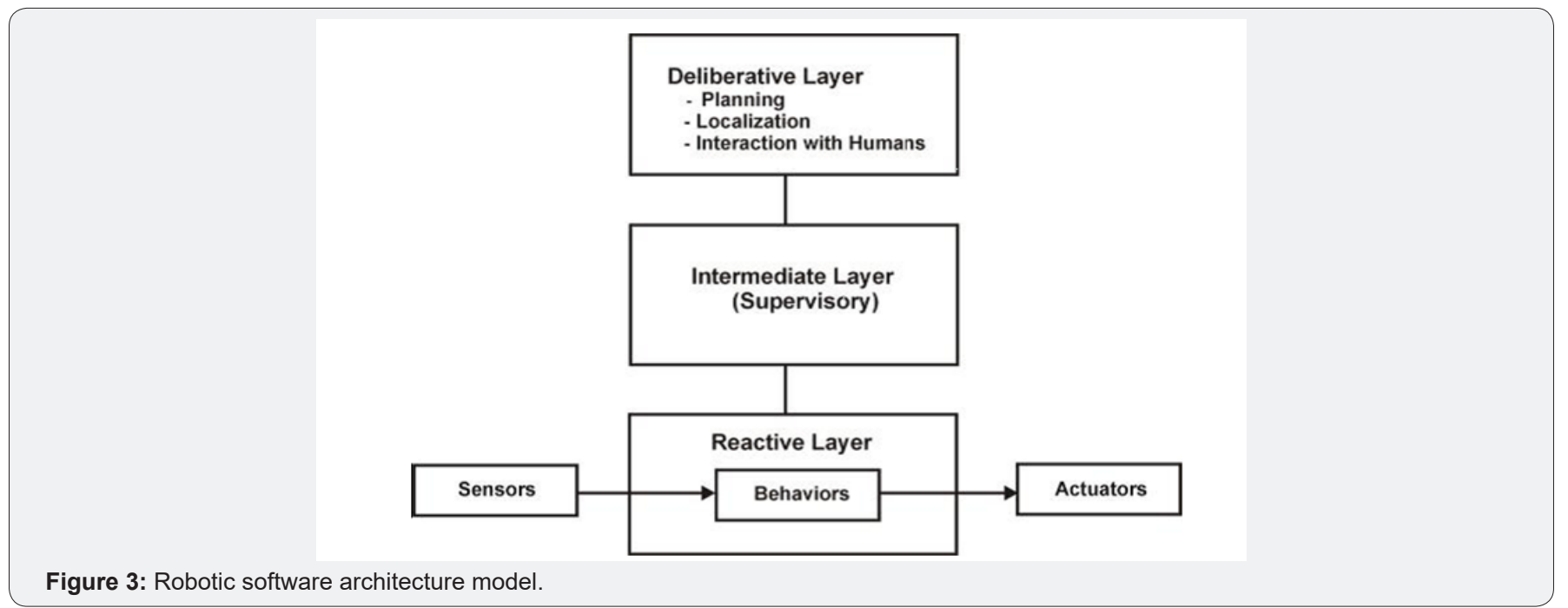

First, let us define risk simply in terms of its opposite, safety: risk is the probability of harm; and (relative) safety is (relative) freedom from risk. Safety in practice is merely relative, not absolute, freedom from harm, because no activity is ever completely risk-free; walking onto one's lawn from inside one's house increases the (however small) risk of death by meteorite strike [6]. Hence, risk and safety are two sides of the usual human attempt to reduce the probability of harm to oneself and others [7].

And finally, some have raised risks of a more abstract sort, indicating the rise of such autonomous robots creates risks that go beyond specific harms to societal and cultural impacts. 
For instance, is there a risk of (perhaps fatally?) Affronting human dignity or cherished traditions (religious, cultural, or otherwise) in allowing the existence of robots that make ethical decisions? Do we 'cross a threshold' in abrogating this level of responsibility to machines, in a way that will inevitably lead to some catastrophic outcome? Without more detail and reason for worry, such worries as this appear to commit the 'slippery slope' fallacy. But there is worry that as robots become 'quasipersons', even under a 'slave morality', there will be pressure to eventually make them into full-fledged Kantian-autonomous persons, with all the risks that entails [8].

\section{Background for Work}

Many people thinking about Spock, the half-Vulcan and half-human character of Star Trek, as the supporter saint of computer science. They highly intelligent, highly rational, highly unemotional, attractive to women etc. A famous image is that Spock didn't have any emotions: after all Spock almost never expressed emotion, excepting his characteristic pronouncement of the word "fascinating" upon thoughtful something new. In fact, as the actor Leonard Nimoy describes in his book the character Spock did have emotion; he was just very good at suppressing its expression. Majority people think that Spock do not having emotion. When someone never used to expresses emotion, it is appealing to think that emotion is not there [9-11]. In affective computing, we can separately examine functions that are not so easily separated in humans. For example, the Macintosh OS exhibits a smile for years upon successful boot-up. But few people would confuse its smile albeit an emotional expression - with a genuine emotional feeling. Machines can take the emotional appearance well mannered, with having dissimilarities in feelings similar to those we would have: they can generate separate expression, gestures and postures from feeling. With a machine it is easy to see how emotion expression does not imply "having" the underlying feeling.

Machines that might really "have" feelings are the key area of affective computing and love affection engineering that coined serious doubt about in 1997 book titled Affective Computing. We think the discussions there, and in a later book chapter on this topic are still timely and will not plan to add to them here. Researchers in the last decade have obtained dozens of scientific findings illuminating important roles of emotion in intelligent human functioning, even when it looks like a person is showing no emotion. These findings possible to restructure, redesign and reengineering with scientific understanding of emotions and can better motivation to work to young researchers with consider that emotional mechanisms might be more valuable than previously believed. Consequently, a number of researchers have charged ahead with building machines that have several affective abilities, especially: recognizing, expressing, modeling, communicating, and responding to emotion. And, insight these domains, various criticisms and challenges have arisen. Present work addresses such matters. The term emotion refers to relations among external incentives, thoughts, and changes in internal feelings, as weather is a super ordinate term for the changing relations among wind velocity, humidity, temperature, barometric pressure, and form of precipitation. In general, a unique combination of these meteorological traits buildup a storm, a tornado, a blizzard, or a hurricane events and that are co-relate to the temporary but intense emotions of fear, joy, excitement, disgust, or anger. But wind, temperature, and humidity vary continually without producing such extreme combinations [12].

\section{Ethics}

With example, if your boss yells at you, is it wrong to detect his angry voice, or to recognize he is angry? Is it unethical, once you've recognized his anger, to try to take steps to alleviate his anger (or to "manipulate" it, perhaps by sharing new information with him, so that he is no longer angry? One can imagine of scenario where the foregoing replies are "no": e.g. He is shouting at you directly, and clearly wants you to recognize it and take steps in response. And, one can imagine the answers might be more complex if you surreptitiously detected his anger, and had nefarious purposes in mind by attempting to change it [13]. Humans routinely scanned, recognize, and respond to emotions using cognitions and manipulating them in ways that most would consider highly ethical and needful. Playing music to cheer up a friend's mood, eating chocolate, exercising to perk one up, and other manipulations count among many that can be perfectly acceptable. That said, unscrupulous uses by people and by people via machine of affect detection, recognition, expression, and manipulation. Some of these, including ways affective machines might mislead customers, assuage productive emotional states, and violate privacy norms, are discussed in Picard and in Picard and Klein [14].

In 1872, Charles Darwin Published a ground breaking bookThe Expression of the Emotions in Man and Animals 2

It was the conclusion about to 34 years of work on emotion and emotional intelligence and resource two important contributions to the field. The first was the notion that animal emotions are homologues for human emotions- a logical extension of Darwin's early works on evolution [15]. Darwin was one of the most promised people who show this by comparing and analyzing countless sketches and photographs of animals and people in different emotional states to reveal cross-species similarities as shown in below figure. .He also proposed that many emotional expressions in humans, such as tears when upset or baring the teeth when angry, are vestigial patterns of action. Darwin second contribution was the proposal which limit to set of fundamental or' basic' emotions are present across species and across cultures including anger, fear, surprise and sadness and all .These two ideas had a profound influence on affective neuroscience by promoting the 


\section{Robotics \& Automation Engineering Journal}

use of research in animals to understand emotions in humans and by giving impetus to a group of scientists who espoused

the view that different basic emotions had separable eneural substrates [16] (Figure 4).
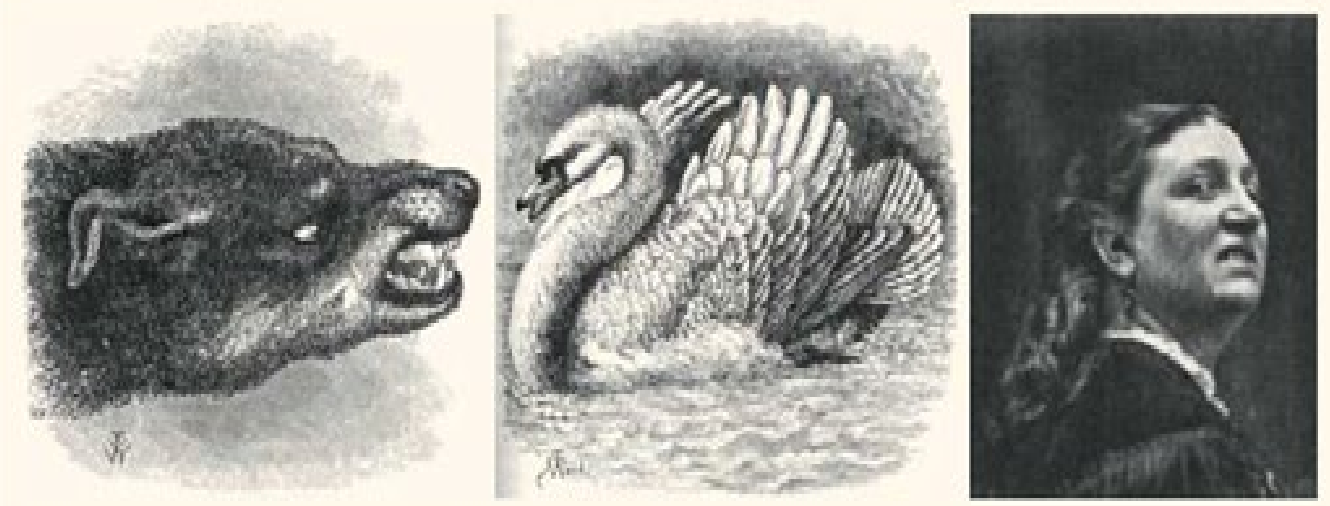

\section{Figure 1 | Darwin's drawings. Drawings and photographs used by Darwin² to llustrate cross-species similarities in emotion expression - in this case, anger/aggression.}

Figure 4: Darwin's Drawings.

\section{Can Robots Have Emotions}

Science fiction is full of machines that have feelings.In 2001: A Space Odyssey, the onboard computer turns against the crew of the spaceship Discovery, and utters cries of pain and fear when his circuits are finally taken apart. In Blade Runner, humanoid robots are distressed to learn that her memories are not real, but have been implanted in her silicon brain by her programmer. In Bicentennial Man, Robin William splays the part of a robot that redesigns his own circuitry so that he can experience the full range of human feelings. These stories achieve their effect impart because the capacity for emotion is often considered to be one of the main differences between humans and machines. This is certainly true of the machines we know today. The responses we receive from computers are rather dry affairs, such as "system error 1378". People sometimes get angry with their computers and shout at them as if they had emotions, but the computers take no notice. They neither feel their own feelings, nor recognize yours [17]. The gap between science fiction and science fact appears vast, but some researcher's inartificial intelligence now believes it is only a question of time before it is bridged. There is huge research in progress in each month in the domain of Affective Computing and an advance result comes in the form of primitive emotional machines. However, some critics argue that a machine could never come to have real emotions like ours. At best, they claim, clever programming might allow it to simulate human emotions, but these would just be clever fakes [18].

\section{What are Emotions}

In humans and other animals, we tend to call behavior emotional when we observe certain facial and vocal expressions like smiling or snarling, and when we see certain physiological changes such as hair standing on end or sweating. Since most computers do not yet possess faces or bodies, they cannot manifest this behavior. However, in recent years computer scientists have been developing arrange of' animated agent faces' [19], programmers that generate images of humanlike faces on the computer's visual display unit. These images can be manipulated to form convincing emotional expressions. Others have taken things further by building three-dimensional synthetic heads. Cynthia Breazealand colleagues at the Massachusetts Institute of Technology (MIT) have constructed a robot called' Kismet' with moveable eyelids, eyes and lips. The range of emotional expressions available to Kismet is limited, but they are convincing enough to generate sympathy among the humans who interact with him. Breazeal invites human parents to play with Kismet on a daily basis. When kismet alone seems to be sad and when with someone company seems to be happy and also able to understand other human expressions and response accordingly. Does Kismet have emotions, then? It certainly exhibits some emotional behavior, so if we define emotions in behavioral terms, we must admit that Kismet has some emotional capacity [20].

Presently Kismet doesn't exhibits full of human like emotional behaviour but seems to be towards developing phase and one day as similar like human beings. Chimpanzees do not display the full range of human emotion, but they clearly have some emotions. Dogs and cats have less emotional semblance to us, and those doting pet-owners who ascribe the full range of human emotions to their domestic animals are surely guilty of anthropomorphism. There is a whole spectrum of emotional capacities, ranging from the very simple to the very complex. Perhaps Kismet's limited capacity for emotion puts 
him somewhere near the simple end of the spectrum, but even this is a significant advance over the computers that currently sit on our desks, which by most definitions are devoid of any emotion whatsoever. As affective computing progresses, we may be able to build machines with more and more complex emotional capacities. Kismet doesn't have voice and feelings in voice mechanism but promising advanced research change this dream into reality soon. Today's speech synthesizers speak in an unemotional monotone. In the future, computer scientists should be able to make them sound much more human by modulating nonlinguistic aspects of vocalization like speed, pitch and volume [21]. Facial expression and vocal intonation are not the only forms of emotional behavior. We also infer emotions from actions. When, for example, we see an animal stop abruptly in its tracks, turn round, and run away, we infer that it is afraid, even though we may not see the object of its fear. For computers to exhibit this kind of emotional behavior, they will have to be able to move around. In the jargon of artificial intelligence, they will have to be "mobots" (mobile robots). In lab at the University of the West of England, there are dozens of mobots, most of which are very simple. Some, for example, are only the size of a shoe, and all they can do is finding their way around a piece of the floor without bumping into anything. Sensors allow them to detect obstacles such as walls and other mobots. Despite the simplicity of this mechanism, their behavior can seem eerily human. When an obstacle is detected, the mobots stop dead in their tracks, turnaround, and head off quickly in the other direction. To anybody watching, the impression that the mobot is afraid of collisions is irresistible. Are these mobots really afraid? Descartes, for example, claimed that animals did not really have feelings like us because they were just complex machines, without a soul. When they screamed in apparent pain, they were just following the dictates of their inner mechanism. Now that we know that the pain mechanism in humans is not much different from that of other animals, the Cartesian distinction between sentient humans and' machine-like' animals does not make much sense [22]. In the same way, as we come to build machines more and more like us, the question about whether or not the machines have 'real' emotions or just 'fake' one swill become less meaningful. The current resistance to attributing emotions to machines is simply due to the fact that even the most advanced machines today are still very primitive. Some experts estimate that we will be able to build machines with complex emotions like ours within fifty years. But is this a good idea? What is the point of building emotional machines? Won't emotions just get in the way of good computing, or even worse, cause computers to turn against us, as they so often do in science fiction?.

\section{Why Give Computers Emotions}

After this long review the point is clear to give emotion in computers could be very useful for a whole variety of reasons. For a start, it would be much easier and more enjoyable to interact with an emotional computer than with today's unemotional machines. Imagine if your computer could recognize what emotional state you were in each time you sat down to use it, perhaps by scanning your facial expression. You arrive at work one Monday morning, and the computer detects that you are in a bad mood. Rather than simply asking you for your password, as computers do today, the emotionallyaware desktops might tell you a joke, or suggest that you read particularly nice email first. Perhaps it has learnt from previous such mornings that you resent such attempts to cheer you up. In this case, it might ignore you until you had calmed down or had a coffee. It might be much more productive to work with a computer that was emotionally intelligent in this way than with today's dumb machines. This is not just a flight of fancy. Computers are already capable of recognizing some emotions [23]. If ran Essa and Alex Pentland, two American computer scientists, have designed a program that enables a computer to recognize facial expressions of six basic emotions. When volunteers pretended to feel one of these emotions, the computer recognized the emotion correctly ninety-eight per cent of the time. This is even better than the accuracy rate achieved by most humans on the same task! If computers are already better than us at recognizing some emotions, it is surely not long before they will acquire similarly advanced capacities for expressing emotions, and perhaps even for feeling them. In the future, it may be humans who are seen by computers as emotionally illiterate, not vice versa.

\section{Modeling}

\section{System error detection-correction model}

Above Figure 5 shows system error detection-correction model which highlighted critical thinking on robotics violence. Basically Ultra Artificial Intelligence with human-like capabilities partly based on two strong segments i.e. UAISoftware and UAI-Hardware to engineer Humanoid. These humanoid mechanisms strongly based on Self Programming Support Unit for self learning using artificial preceptor with intention to implement self sensation, actuation, meaning memories generation and execute like mankind. Hence using sensors, transducers, motors and actuators mechanism with NLP, Image Processing software possible to interact with external environment. Since Humanoid scan \& learn from environment there might possible sources hidden in environment for diversification humanoid for violence or undesired execution or use I called them "Triggers". There are two triggers possible for robotics violence one is "internal violence triggers" and another is "external violence triggers". In internal violence triggers self bad conduct by self programming responsible for it which boost to system error 1378 occurred due to internal reasons. Whereas when self programming support unit hike, hijacked, in control of enemies or corrupted through virus programming to change good ethics into bad ethics of humanoid caused robotic violence which boost to system error 1378 occurred due to external reasons [24]. 


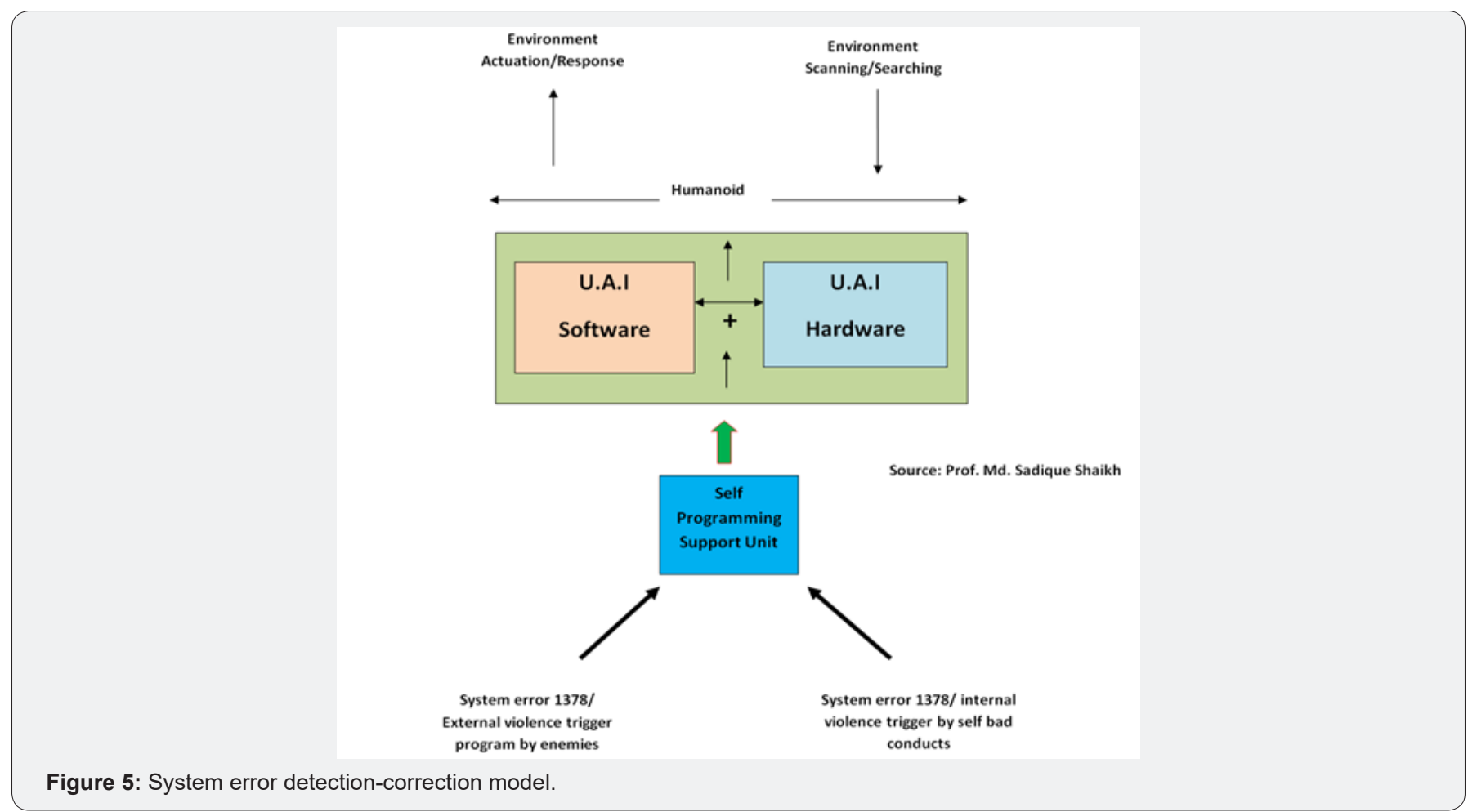

\section{Cause-effect model}

(Figure 6) This is the second purposed model in effect of first model named Cause-Effect model. In this model had given brief but lucid knowledge about Humanoid violence causes and their effects on world, technology and ultimately mankind civilization. As I said in first model discussion there are two robotics violence triggers viz. internal \& external triggers and this cause effect to "self distractive program with bad ethics" due to this cause system error 1378 occurred in effect which further cause to "Humanoid-Human Wars (Humanoid/Robotics Violence)" this effect will further become cause humanoid against of human for love, affection, emotion, respect and rights .i.e. world either will end or ruled by Humanoid machines over Human due to high intelligence and processing abilities.

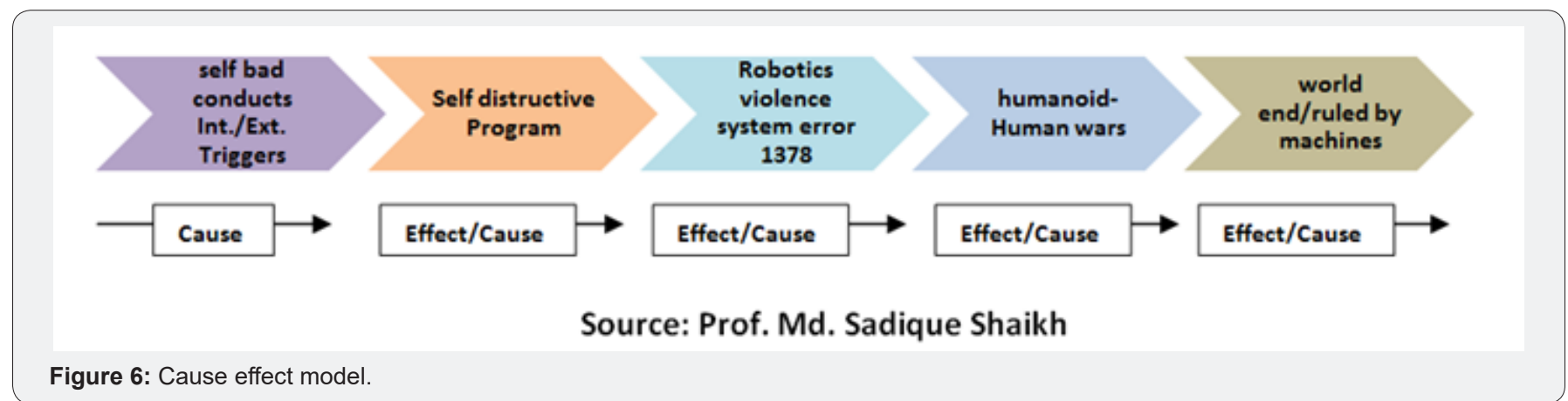

\section{Human friendly humanoid designing model}

This is my last model to exhibit idea how can how Human friendly Humanoid engineering possible instead of only going technically very high very we must have to think could we able to control that technology if it itself become against of us, is there we have any provision in our advanced AI engineering to stop to do so. I have suggested some one can enhance it further; these are

1) If system error 1378 occurred make immediate \& emergency replacement of chip in standby to avoid robotics violence.
2) Engineer constructive counseling program in self programming support unit to trace and tackle with system error 1378 with self killing of malfunction and execution to avoid robotics violence.

3) Implement system error 1378 monitoring \& security program as individual component to avoid robotic violence,

4) I strongly recommend available inbuilt subroutine to activate and direct self destructive programs which make Humanoid hardware death after its execution which trigger with inbuilt command "self destroy" when system error 1378 traced to avoid robotics violence (Figure 7). 
Figure 7: Human friendly humanoid designing model.

\section{Conclusion}

Relational agents, as any technology, can be abused. Agents which earn our trust overtime can be used to provide more potent means of persuasion for marketers than more passive forms of advertising. If eventually come to rely on our agents as sources of grounding for our beliefs, values and emotions (one of the major functions of close human relationships) then they could become a significant source of manipulation and control over individuals or even over entire societies. There are those who also feel that any anthropomorphic interface is unethical, because it unrealistically raises users' expectations. One way to combat this problem is through proper meta-relational communication-having the agent is as clear as possible about what it can and can't do, and what expectations the user should have about their respective roles in the interaction anti violence robotic programmes must need to develop before to give human like emotions to Humanoid robots to avoid system error 1378 .

\section{References}

1. Aarup M, Arancli MM, Paned Y, Stader, Stokes 109941 CIPTIMLIMA1V: A inosolodge Lowed planning and for spacecraft AlV. In: Fox M, Zwcben $M$, editors. Knowledge Based Scheduling. Morgan Kauf-mann.

2. Abney S (2007) Semisuperpised Learning for Computational Linguistics.CRC Pins

3. Abramson B, Yang M (1989) Divide and corquer under global constraints: A solution to the N-queens problem. I. Parallel mul Dintributea COPE paring, 6121, env) 6(3): 649-662.

4. Acalioptas D (2009) Random satisfiability. In: Siam A, Heige M, Assl Marren H, Walsh TL, editors. A Handbook of Sonsfirreildy IOS Press.

5. Achlloptas D, Bernie P, Molloy M (2004) Exponential bounds for DPLL below die Liy threshold. In: SODA-64.

6. Bacchus R, Grove A, Halpern IY, Koller 0.119'921. From satisfies to beliefs. In: AMMO, pp: 602-608.

7. Bacchus F, van Beek P (1998) On the conversion between non-binary and binary constraint sans-fucuimo gi olden. In: AAAL4R, pp: 311-315.

8. Bacchus F, tin Rim P (1995) Dynamic vain-able ordering in CS9s. In: CP-95, pp: 258-275.
9. Bachmann PGH (1894) Die analytische Zahtem theorie BG Teal: user, Leiptig.

10. Chamlak E (1996) Tern-bank grammars. In: AAV.96: 1031-1036.

11. Chamlak E (1997) Statistical parsing with a context-free grammar and word statistics. In: AAA!. 97: 598-6d3.

12. Charnlak E, Goldman RI (1992) A Bayesian model of plan recognition. ALI 64411 64(1): 53-79.

13. Charialak E, McDermott D (1985) Intraday-lion to Arnficial Intelligence. Addison-Wesley. Chandra E, Riesbtol C, McDermott D, i91471 Pre,ram. Ming (2nd edn). Lawrence Erlbaarn Associates.

14. Chamialt E (1991) Bayesian networks without /CATE. AlMug, EJ(d), pp: 55-63.

15. Sadique Shaikh Md (2013) Analysis and modeling of Strong A.I to engineer BIONIC brain for humanoid robotics application. In American Journal of Embedded System and Applications, Published by Science Publishing Group. New York, America 1(2).

16. Sadique Shaikh Md (2013) Ultra artificial Intelligence(UAI): Redefining AI for new research dimension. In International Journal of Engineering and Scientific Research(IJESR), USA 2(1): 57-68.

17. Sadique Shaikh Md (2014) Fundamental Engineering to Design and Implement Facial Expression, Emotions Recognition and Artificial Emotional Intelligence (AEI) in Humanoid Robotics. Computer Science and Applications (CSA), Ethan Publishing, USA 1(2): 102-112.

18. Sadique Shaikh Md (2017) Ultra Artificial Intelligence (UAI): Re-define AI for New Research Dimension. in Advanced Robotics \& Automation (ARA), OMICS International, London 6(2): 1-3.

19. Sadique Shaikh Md (2017) Fundamental Engineering for BrainComputer Interfacing (BCI): Initiative for Neuron-Command Operating Devices. In Computational Biology and Bioinformatics (CBB), Science PG, USA 5(4): 50-56.

20. Sadique Shaikh Md (2018) Defining ultra artificial intelligence (UAI) implementation using bionic (biological-like-electronics) brain engineering insight. MOJ App Bio Biomech, USA 2(2):127-128.

21. Sadique Shaikh Md (2018) Insight Artificial to Cyborg Intelligence Modeling. Arch Ind Engg, USA 1(1): 1- 5.

22. Sadique Shaikh Md (2018) Insight Bionic Brain Engineering. Journal of Biotechnology and Bioengineering, India 2(1): 1-3. 
23. Sadique Shaikh Md (2018) Insight Natural Vision Processing like Engineering for Machine Vision. In Journal of Dynamics of Machines, Mach, London, UK 1(1): 1-4.

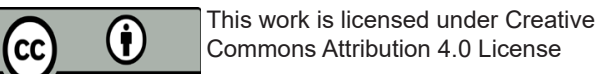

BY DOI: 10.19080/RAEJ.2018.03.555610
24. Sadique Shaikh Md (2018) Medical Robotics. In Case Rep Surg Invasive Proced London, UK 2(2).

\section{Your next submission with Juniper Publishers} will reach you the below assets

- Quality Editorial service

- Swift Peer Review

- Reprints availability

- E-prints Service

- Manuscript Podcast for convenient understanding

- Global attainment for your research

- Manuscript accessibility in different formats

( Pdf, E-pub, Full Text, Audio)

- Unceasing customer service

Track the below URL for one-step submission https://juniperpublishers.com/online-submission.php 